\title{
Anti-fingerprint glass surface created by mechanical removal process
}

\author{
Kazuma SHIBATA*, Yuta NAKASHIMA** and Yoshitaka NAKANISHI** \\ ${ }^{*}$ Graduate School of Science and Technology, Kumamoto University \\ Kurokami 2-39-1, Chuo-ku, Kumamoto 860-8555, Japan \\ **Faculty of Advanced Science and Technology, Kumamoto University \\ Kurokami 2-39-1, Chuo-ku, Kumamoto 860-8555, Japan \\ E-mail: y-naka@mech.kumamoto-u.ac.jp
}

Received: 28 July 2019; Revised: 15 October 2019; Accepted: 6 December 2019

\begin{abstract}
The use of various types of information devices equipped with touch panels has been increasing. When these devices are used directly with fingers, the devices become dirty owing to sweat and oil from users. Therefore, there is considerable interest in keeping the surfaces of devices free from fingerprints and dirt. Antifouling films and coatings are being developed for this purpose. In addition, a method of changing the surface shape by adding a microstructure to a material surface has been developed. The purpose of this study is to add the antifouling function to glass surfaces using a mechanical removal process. We attempted to change the surface shape by processing a material surface directly. The processing method was microslurry-jet (MSJ) processing, which is a mechanical removal method. In addition, a masking process was used to create microsurface structures. As an example, we evaluated the anti-fingerprint glass created by these processing methods. Microsurfaces were successfully created on the glass surface through the combination of the masking process and MSJ processing. Moreover, the visibility of the created glass, surface characteristics, friction characteristics, and the adhesion of stains were evaluated. With respect to visibility, surface characteristics, and adhesion, it was possible to observe the changes in surface characteristics due to the microsurfaces. With regard to friction characteristics, differences in surface patterns were found for individual subject data.
\end{abstract}

Keywords : Anti-fingerprint, Glass, Microstructure, Mechanical removal process, Microslurry-jet

\section{Introduction}

The use of various types of information devices equipped with touch panels has been increasing. When these devices are used directly with fingers, the devices becomes dirty owing to sweat and oil from users (Uhlmann et al., 2007). Therefore, there is considerable interest in keeping the surfaces of devices free from fingerprints and dirt. Antifouling films and coatings are being developed for this purpose (Kesmez et al., 2017). In addition, a method of changing the surface shape by adding a microstructure to the material surface has been developed (Jheng and Lee, 2016; Saito et al., 2008; Tokuhisa et al., 2017). Microsurface structures are created by a chemical removal process by etching or removal by laser processing (Clavería et al., 2019). However, such chemical removal processing is limited by materials because chemical solutions are used (Saito et al., 2008). Laser processing removes a surface with a strong force. As a result, the surface becomes rough and the substrate becomes damaged, and this affects touch. This study has explored a processing method that can solve these problems. Microslurry-jet (MSJ) processing, which is a mechanical removal method, was considered because it can be applied to numerous materials, it can be processed on the order of nanometers in the vertical direction, and it provides a continuous curved surface after processing (Nakanishi et al., 2018; Yin et al., 2018). In addition, a masking process was used to create microsurface structures. Glass was used as the material in this study. Glass is a brittle material, and it is broken, scratched, or easily soiled during mechanical removal. It was shown that these problems can be addressed, and the anti-fingerprint glass produced using the above processing method was evaluated. 


\section{Materials and methods}

\subsection{Strategy for designing anti-fingerprint glass surface}

In this study, flat glass was used as the object to be processed. The glass comprised of $\mathrm{SiO} 2$ with $13 \% \mathrm{Na} 2 \mathrm{O}$ and $10 \% \mathrm{CaO}$. The anti-fingerprint surface was created by reducing the actual contact area between the finger and glass (Fig. 2). When the height of a shape created on a glass surface is large, the resistance due to the plowing effect is large and visibility is poor (Jheng and Lee, 2016; Wang et al., 2010; Kamminga and Janssen, 2007). When the height of a convex shape is small, the effect of reducing the contact area is not observed (Zhou et al., 2018). Convex pitch and diameter are considered to be the same. In this work, three types of pattern were prepared by changing the diameter and interval to narrow down the parameters.

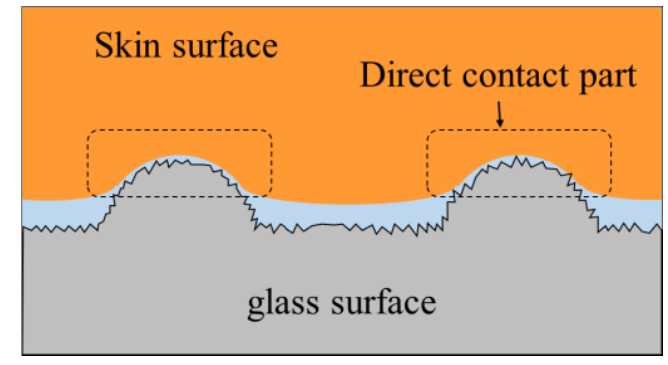

Fig. 1 Schematic of contact area of finger and processed glass.

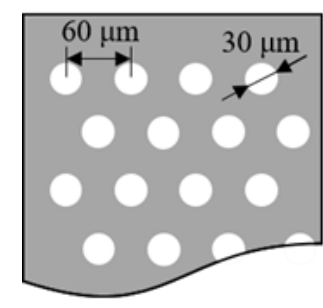

Type-A

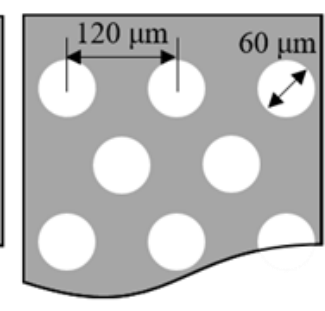

Type-B

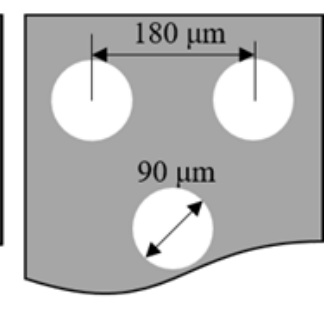

Type-C
Fig. 2 Schematic of pattern design.

\subsection{Glass created by a mechanical removal process}

A masking process based on the microelectromechanical systems technology was adopted to create the antifingerprint glass (Fig. 3) with various microstructures (Al-Milaji and Zhao 2017). Before the masking process, the glass was ultrasonically cleaned using methanol, acetone, and ultrapure water. Next, the organic compounds and other contaminants on the glass surface were removed using piranha solution (a mixture of 3:1 sulfuric acid, 30\% hydrogen peroxide, and up to $40 \% \mathrm{HF}$ ) (Feitosa et al., 2017). The SU-8 photocurable resin was used as a photoresist (Saha et al., 2009; Psoma et al., 2010; Ginestra et al., 2019). It was dipped in silane coupling material (kbm 403 ) for 5 min to increase the bonding strength of glass (inorganic material) and SU-8 (organic material) (Psoma et al., 2010; Hussain et al., 1996; Baba et al., 2019). The silane coupling agent contains two or more different reactive groups. One reactive group is capable of chemically bonding with an inorganic material, and the other is capable of chemically bonding with an organic material. The surface of the processed glass was coated with SU-8. It was irradiated with ultraviolet rays through a photomask after drying for $12 \mathrm{~h}$ at room temperature $\left(20\right.$ to $\left.25^{\circ} \mathrm{C}\right)$. The nonirradiated parts were removed through immersion in 2-acetoxy-1-methoxypropane. The irradiated parts were retained, and the patterns were created.

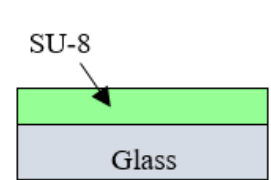

1.Coating

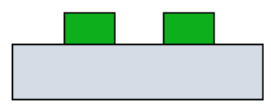

4.Pattern on surface

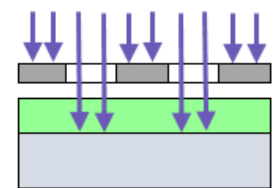

2.Ultraviolet irradiation

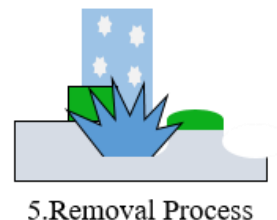

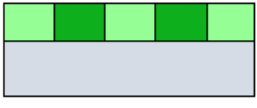

3.Resist development

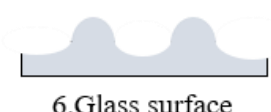

Fig. 3 Method of creating anti-fingerprint glass by masking process and microslurry-jet processing. 
Next, MSJ processing was used instead of the chemical removal process. MSJ processing allows for precise texture processing. When switching to mechanical removal such as MSJ, surface processing can be performed on various materials that are difficult to etch. It has ultraprecise machining capability of the order of nanometers in the orthogonal direction on a material. It is a wet blasting technique that uses alumina particles as an abrasive medium with compressed air and water to produce microstructures. A slurry consisting of pure water with $3.2 \mathrm{wt} \%$ alumina particles was prepared. Micropolyhedral alumina particles were used, and their average diameter was $1.2 \mu \mathrm{m}$ (WA \# 8000). The slurry was sprayed vertically on the glass surfaces through an injection nozzle with a square shape of $1 \mathrm{~mm}$ width, with compressed air at $0.3 \mathrm{MPa}$. The injection nozzle could be moved parallel to the processed surface using a numerical control system. The nozzle was moved to achieve a feed rate of $3 \mathrm{~mm} / \mathrm{s}$ and a feed pitch of $0.1 \mathrm{~mm}$. After finishing a reciprocal movement on a surface, the movement direction of the nozzle was changed by $90^{\circ}$ to finish another reciprocal movement on the surface. MSJ processing was repeated for up to 8 cycles (Baba et al., 2019).

\subsection{Evaluation of anti-fingerprint surface created by a mechanical removal process}

The surfaces obtained after the masking process and MSJ processing were observed using a confocal laser microscope (LEXT OLS 4100, Olympus, Japan). The shapes of the surfaces were evaluated numerically and by utilizing images.

After processing, it was verified whether the glasses retained permeability. The glasses were placed on the backlight of a smartphone to verify visibility (Fig. 4).

As the shape of the glass surface changed, the hydrophobicities of the normal glass and processed glasses were compared. The contact angle was measured using the droplet method (Tokuhisa et al., 2017; Kamminga and Janssen, 2007). Before measurement, all glasses with or without the MSJ processing were cleaned in an ultrasonic cleaner using ethanol, acetone, and ultrapure water. The contact angle was the average of 10 measured values. The following formula for the convex ratio was used to obtain the values of the height and area of the shapes:

\section{Convex ratio $=\sum \pi\left(\mathrm{d}_{\mathrm{h} / 2} / 2\right)^{2} /($ AOI: Area of Interest)}

Three males participated in the experiment (22-24 years). All procedures were in accordance with the 1964 Helsinki declaration and its later amendments or comparable ethical standards. The ethical approval for the tribological testing was obtained from the ethical review committee at Faculty of Advanced Science and Technology, Kumamoto University, Japan. Informed consent was obtained from all participants.

The contact/friction between the glass surface after the MSJ processing and the forefinger (second finger) skin was analyzed. Figure 5 shows the experimental apparatus for the friction test used to verify fingerprint adhesion. The surface of the forefinger was cleaned before the experiment. The finger was washed with ethanol and pure water and dried for $30 \mathrm{~s}$. Then, the finger was placed on the glass surface, and a load of $1.98 \mathrm{~N}$ was applied on the finger in the vertical direction with respect to the glass. After maintaining this state for $20 \mathrm{~s}$, the finger and glass were separated. The residual status of the fingerprint was observed from the bottom of the glass using a CCD camera.

When measuring friction, the glass was fixed to a stage and an actuator was operated to move the glass on the stage at a constant speed. The stage could translate along the $\mathrm{x}$ axis. An adjustable part was designed to support the participants' fingers to ensure the correct position of the fingers relative to the glass and for the comfort of the participants. The angle between the finger and glass surface was approximately $20^{\circ}$ (Messaoud et al., 2016). A subject's forefinger was brought into contact with the glass and assigned a predetermined vertical load and sliding speed. Subjects were only required to maintain their fingers in their original positions while the stage with the glass was moved by the actuator. The sliding distance was $15 \mathrm{~mm}$. The vertical loads applied in this test were $0.98 \mathrm{~N}, 1.96 \mathrm{~N}$, and $2.94 \mathrm{~N}$. In the reports on the friction between artificial materials and skin, the load zone where the surface shape and friction characteristics are likely to appear was selected and 3 loads were chosen. The stage was moved at a speed of $1.34 \mathrm{~mm} / \mathrm{s}$. This speed was set such that friction behavior was easily measured and the stage moved smoothly. The glasses with and without processing were used in the experiment. Data were recorded at a sampling frequency of $500 \mathrm{~Hz}$. A strain gauge was used to calculate the value of the friction coefficient during relative motion. The actuator was operated by setting the pulse directly using a function generator to reach a frequency that did not resonate with the device. 


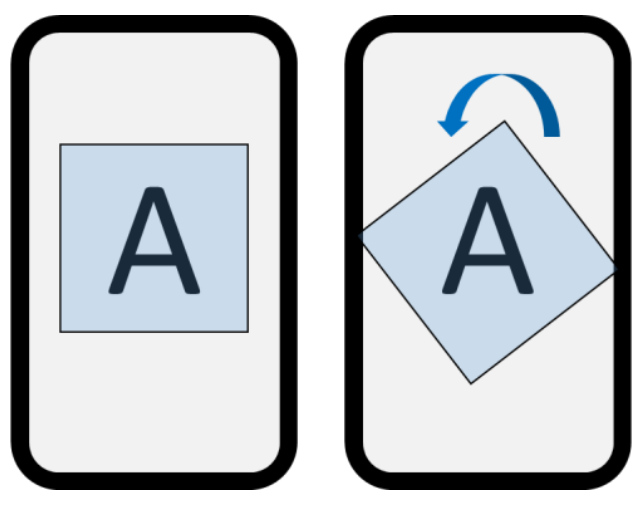

Fig. 4 Schematic of visibility verification.

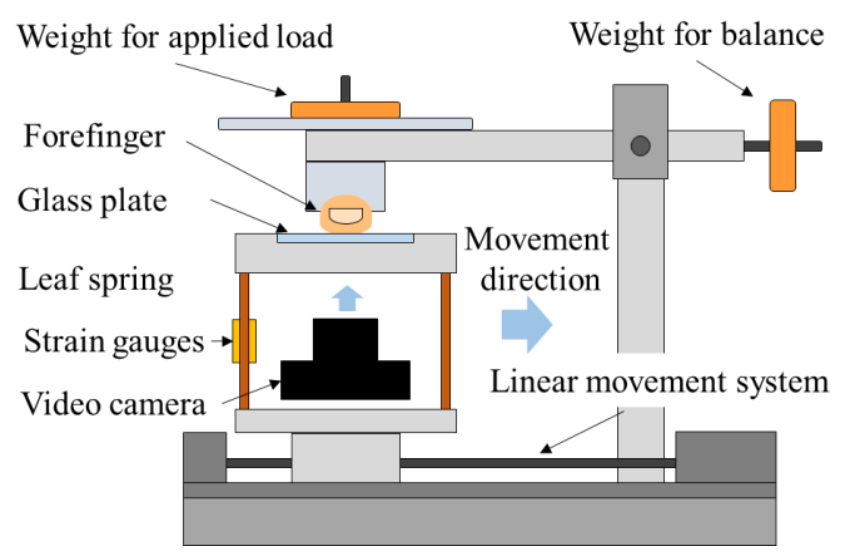

Fig. 5 Device for measuring relative motion state between the human forefinger skin and glass surface.

\section{Results and discussion}

\subsection{Laser microscopic observation}

Figure 6 shows the observation results of the glass after the masking process, which were obtained using a confocal laser microscope. The photoresist was precisely patterned to Type-A (diameter: $30 \mu \mathrm{m}$; pitch: $60 \mu \mathrm{m}$ ), Type-B (diameter: $60 \mu \mathrm{m}$; pitch: $120 \mu \mathrm{m}$ ), and Type-C (diameter: $90 \mu \mathrm{m}$; pitch: $180 \mu \mathrm{m}$ ). The heights of all patterns were $15 \mu \mathrm{m}$ (with \pm 0.2 $\mu \mathrm{m})$. Figure 7 shows the observation results of the glass after the MSJ processing, which were obtained using a confocal laser microscope. A continuous curved convex structure was created on the glass surface after the MSJ processing. In optical microscopy, the glass after the MSJ processing was not clouded. It is considered that the difference between the heights of the patterns after the masking process is due to the difference between the removal rates of SU-8 and glass. During the MSJ processing, SU-8 received vertical and diagonal forces owing to the large nozzle size of the MSJ machine. Therefore, Type-A with a smaller diameter became conical, and it was removed from the top and outer ring. Type-C with a larger diameter had a trapezoidal shape because it was eliminated faster from the top. A missing pattern was observed after the MSJ processing. As Type-A had a small pattern diameter, the adhesion between the glass and SU-8 was low. Therefore, during the masking and MSJ processing, SU-8 separated from the glass surface at certain locations. In this study, SU-8 was tested as a masking resin. In this experiment, the pattern size was limited to a diameter of $30 \mu \mathrm{m}$. However, for example, by changing the characteristics of the masking resin, compatibility with glass is improved and wear resistance is improved. Hence, the pattern size can be reduced.
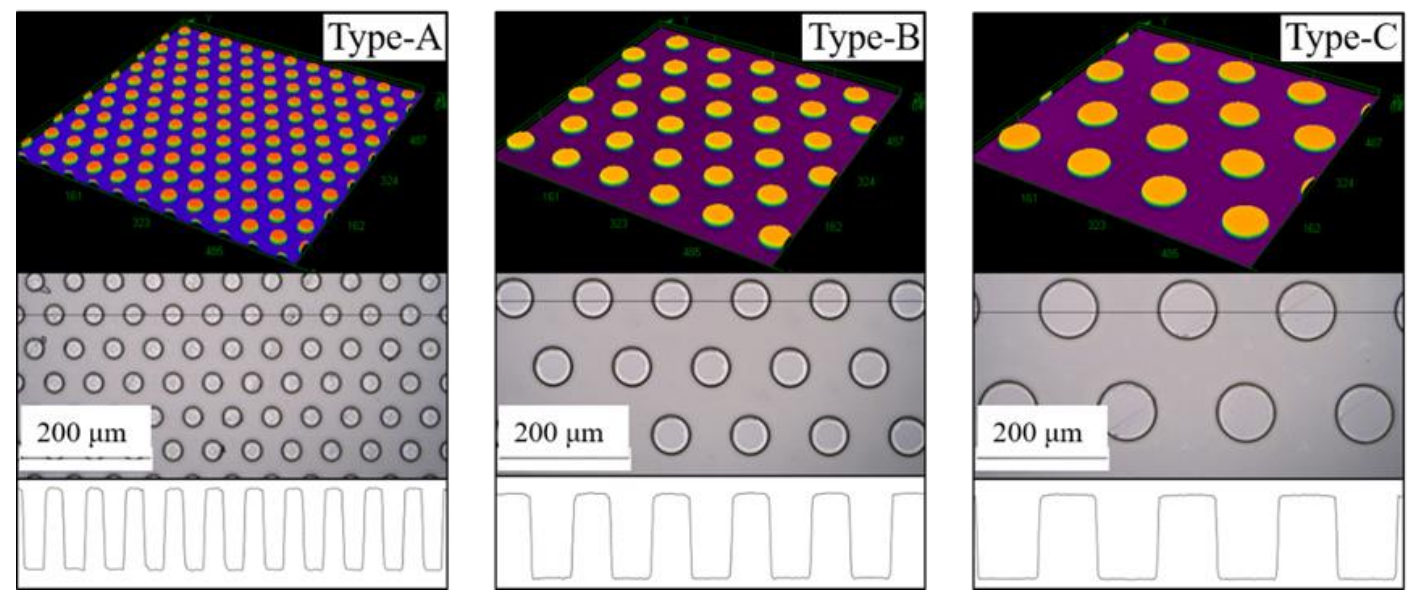

Fig. 6 Observation results of the glass after the masking process, obtained by a confocal laser microscope. 

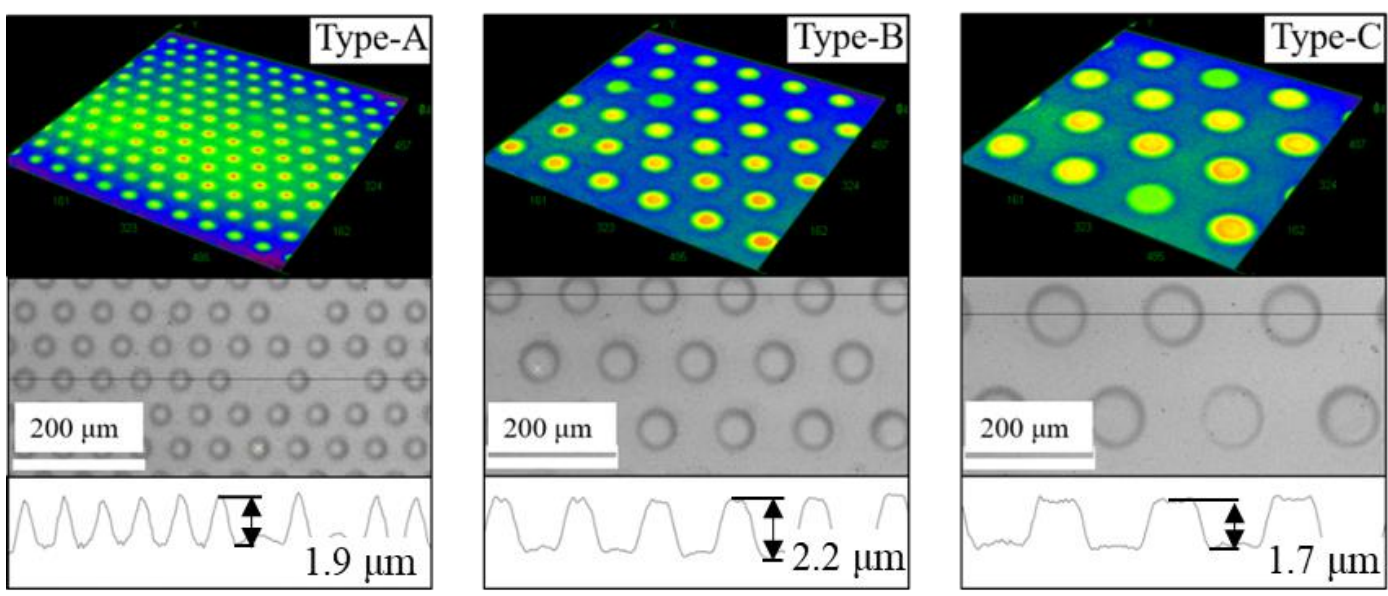

Fig. 7 Observation results of the glass after the MSJ processing, obtained by a confocal laser microscope.

\subsection{Visibility}

Fingerprint adhesion was compared to verify visibility. Comparison images are shown in Fig. 8. A backlight was operated when the touch panel was used. The visibility of the processed glass was verified using a smartphone backlight. The three patterns were not observed when there was a backlight. Interference of light was observed for Type-C, as shown in Fig. 9, but not in Type-A and Type-B. This phenomenon is considered to be caused by the size of this pattern.

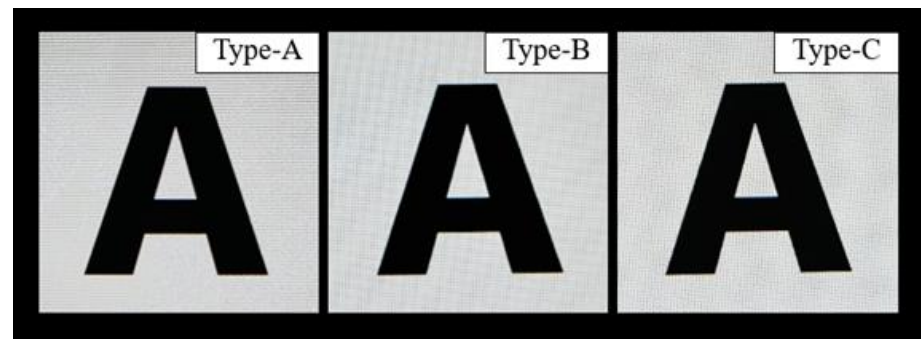

Fig. 8 Change in visibility through the backlight.

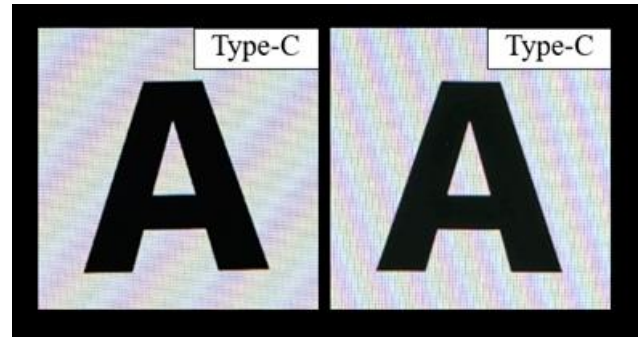

Fig. 9 Interference of light observed for Type-C.

\subsection{Contact angle of water}

As the frictional behavior between the finger skin and artificial material is affected by the hydrophilicity/hydrophobicity of the artificial material, it is considered that the dirt of sebum also influences hydrophilicity/hydrophobicity. Thus, the contact angle was measured. The contact angles of water and the convex ratio are shown in Table 1. The contact angle of the normal glass was $33.5^{\circ}$. The processed glass showed a high contact angle as compared to the normal glass. The contact angle was higher when the size of the pattern was smaller.

The contact area varied depending on the size of the pattern. The convex ratio was calculated and compared using the images obtained via laser observation (Fig. 10). The convex ratio decreased with the pattern size.

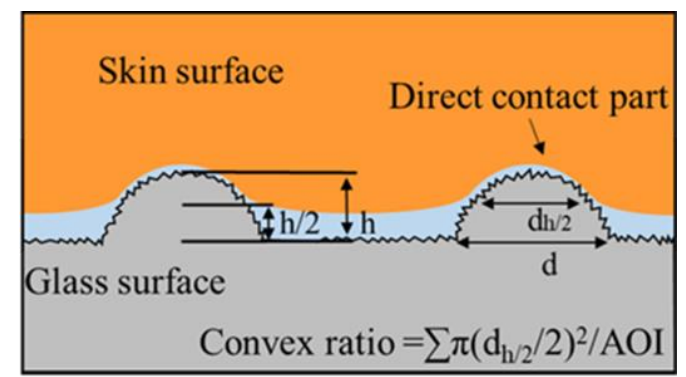

Fig. 10 Schematic of convex ratio. 
Table 1 Contact angle and convex ratio.

$\begin{array}{lll}\text { Type-A } & \text { Type-B } & \text { Type-C }\end{array}$

\begin{tabular}{c|c|c|c}
\hline & Type-A & Type-B & 50.09 \\
\hline Contact angle $\left(^{\circ}\right)$ & 58.87 & 3.03 & 2.62 \\
\hline SD & 2.70 & 0.146 & 0.163 \\
\hline Convex ratio & 0.138 & & \\
\hline
\end{tabular}

\subsection{Fingerprint}

The adhesion of fingerprints was verified when the fingers were resting on the glass surface. Figure 11 shows the experimental results obtained for the four types of glass with the three male participants. Fingerprints were observed throughout the normal glass. The pattern fragmented the fingerprints. Therefore, the glass with a pattern was less visible compared to normal glass. The fingerprint on the glass with a pattern was difficult to confirm. These results might have been obtained because sebum was less likely to adhere as the convex ratio was smaller and the contact angle was higher.

Tester-1 Tester-2 Tester-3

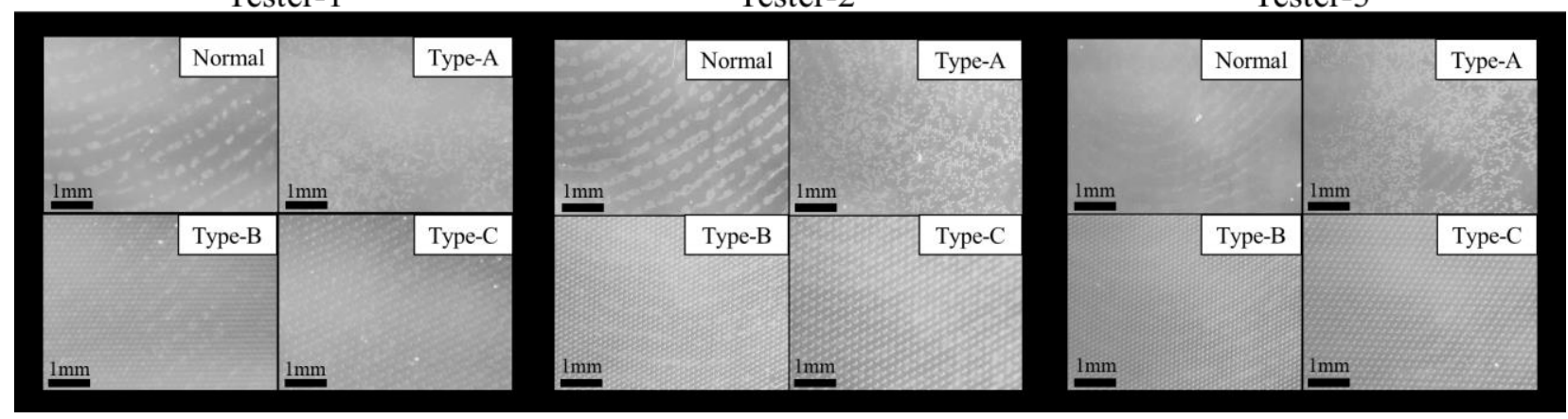

Fig. 11 Fingerprint attachment comparison.

\subsection{Friction}

Figure 12 shows the variation in the coefficient of friction of the forefinger skin for each type of glass. The normal glass and the processed glasses with the three patterns were compared under three different load conditions. At low load, the coefficient of friction tended to be high, and its value was over 1 . These phenomena were consistent with the friction characteristics of soft materials with high cohesion. The friction behavior was almost the same as that of the normal glass. However, a slight vibration presumed to be stick-slip was confirmed. The frequency of vibration and the mean coefficient of friction tended to increase with the pattern size. This phenomenon was considered to be caused either by the digging friction due to the convex structure on the glass surface or by an increase in adhesion force due to an increase in the contact pressure at the convex portion.

Next, we analyzed the experimental video for the representative data of microvibration. The results are shown in Fig. 13. Images were divided every $0.025 \mathrm{~s}$. Considering the fingerprints at the time when the fluctuation appeared in the graph at a load of $2.94 \mathrm{~N}$ for Type-C, it was confirmed that the movement distance of the fingerprints increased. The part from 7.00 to $7.06 \mathrm{~s}$ was considered to be a slip region because the amount of finger movement was large. The other areas were stick regions. It has been reported that a high cohesion force is observed in finger friction, and therefore, the coefficient of friction increases at light loads (Zhang et al., 2105). The processed glass is more likely to stick to the skin compared to the unprocessed glass. Thus, stick and slip are likely to occur during the relative movement process. 


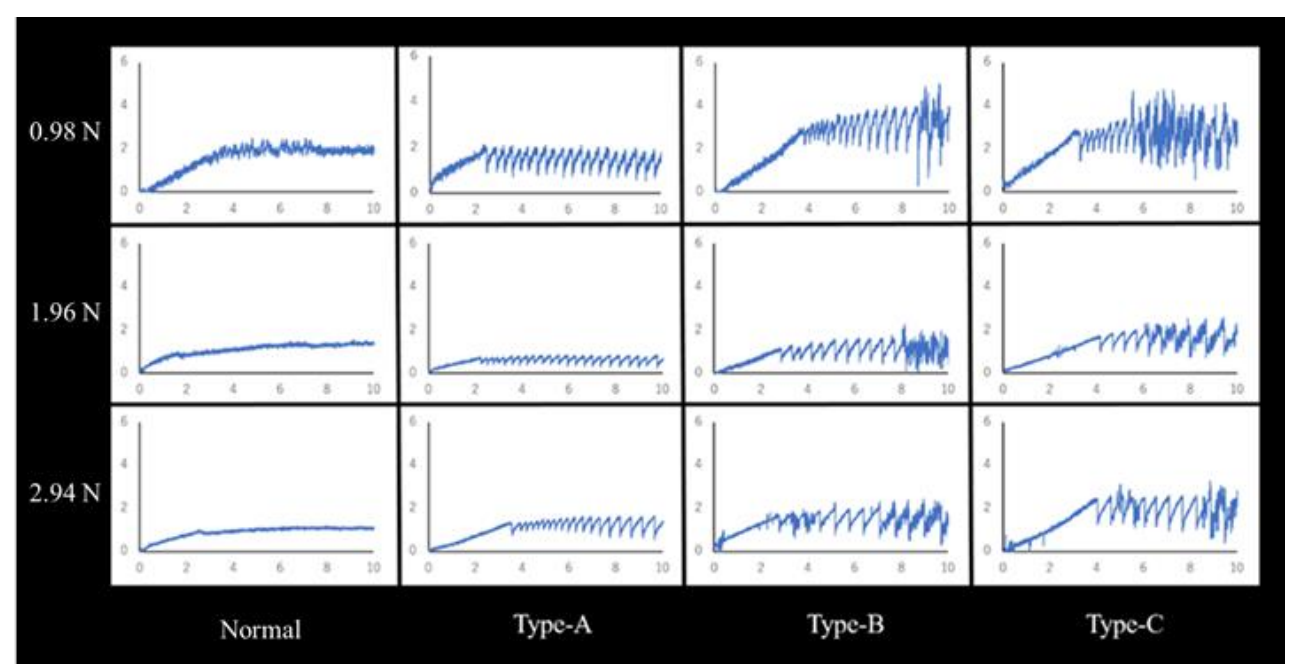

Fig. 12 Change in coefficient of friction (vertical axis) with time (s; horizontal axis).

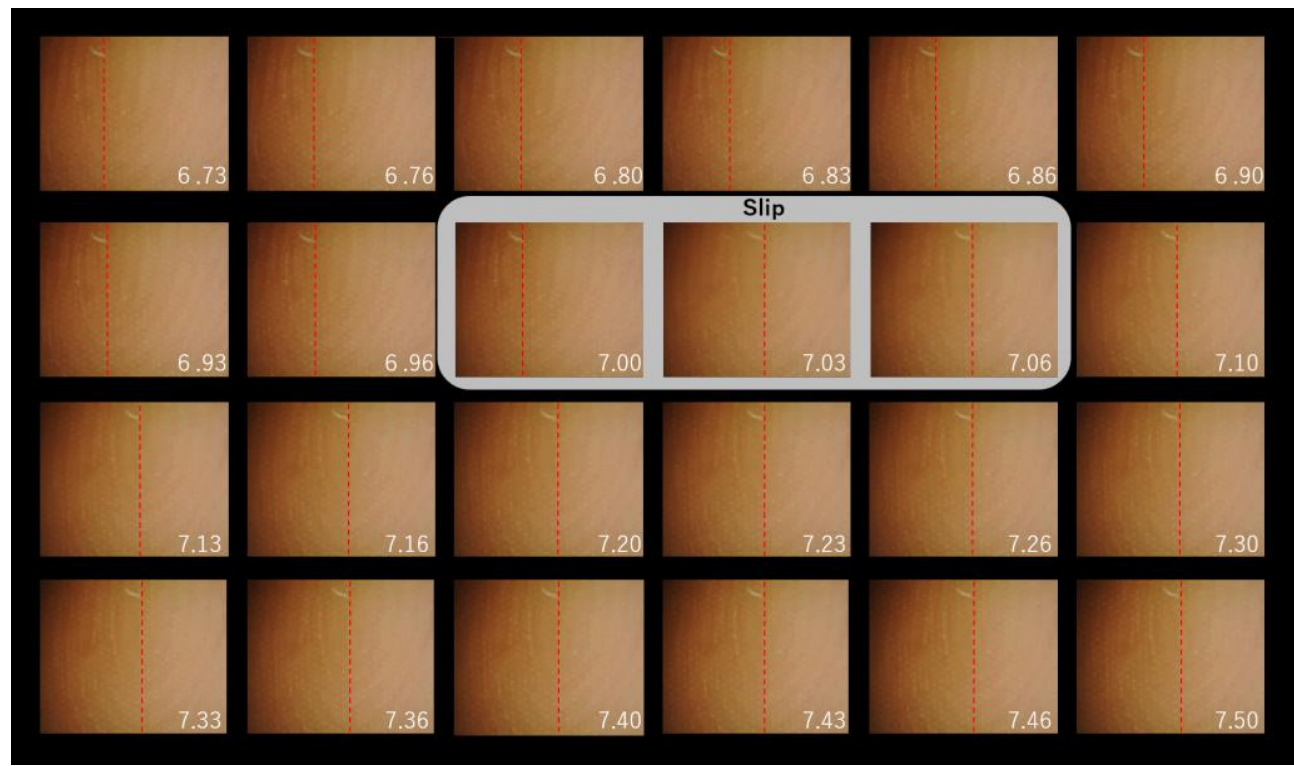

Fig. 13 Video analysis during friction test (load of $2.94 \mathrm{~N}$ for Type-C).

The red line shows a point with a fingerprint.

\section{Conclusion}

The purpose of this study was to add the antifouling function to glass surfaces through mechanical removal. Microsurfaces were created on a glass surface through the combination of a masking process and MSJ processing. Moreover, the visibility of the created glass, surface characteristics, friction characteristics, and the adhesion of stains were evaluated. With respect to visibility, surface characteristics, and adhesion, it was possible to observe the changes in surface characteristics due to the microsurfaces. The pattern with the smallest size was shown to be optimal. However, owing to the compatibility (adhesiveness) between the glass the mask material, the pattern size was limited to $30 \mu \mathrm{m}$. A smaller size may show more optimal characteristics. With regard to friction characteristics, the differences in surface patterns were found for individual subject data. However, it was not possible to perform a universal assessment for all subjects. Therefore, it is necessary to devise an evaluation method and an experimental method that can be applied to all types of data. We plan to conduct investigations with specific application examples. We must include usability evaluation items as statistical evaluation and other important evaluations. 


\section{References}

Al-Milaji, K. N. and Zhao, H., Fabrication of superoleophobic surfaces by mask-assisted electrospray, Applied Surface Science, Vol.396, (2017), pp.955-964.

Baba, T., Nakashima, Y., Takahashi, S., Matsubara, T., Yin, L. and Nakanishi, Y., Micro slurry-jet for surface processing of dental ceramics, Biosurface and Biotribology, Vol.5, No.1 (2019), pp.8-12.

Clavería, I., Elduque, D., Lostalé, A., Fernández, A., Castell, P. and Javierre, C., Analysis of self-lubrication enhancement via PA66 strategies: Texturing and nano-reinforcement with $\mathrm{ZrO} 2$ and graphene, Tribology International, Vol.131, (2019), pp.332-342.

Feitosa, S. A., Lima, N. B., Yoshito, W. K., Campos, F., Bottino, M. A., Valandro, L. F. and Bottino, M. C., Bonding strategies to full-contour zirconia: Zirconia pretreatment with piranha solution, glaze and airborne-particle abrasion, International Journal of Adhesion and Adhesives, Vol.77, (2017), pp.151-156.

Ginestra, P. S., Madou, M. and Ceretti, E., Production of carbonized micro-patterns by photolithography and pyrolysis, Precision Engineering, Vol.55, (2019), pp.137-143.

Hussain, M., Nakahira, A., Nishijima, S. and Niihara, K., Effects of coupling agents on the mechanical properties improvement of the TiO2 reinforced epoxy system, Materials Letters, Vol.26, No.6 (1996), pp.299-303.

Jheng, Y.-S. and Lee, Y.-C., Fabrication of micro/nano hierarchical structures with analysis on the surface mechanics, Applied Surface Science, Vol.384, (2016), pp.393-399.

Kamminga, J. D. and Janssen. G. C. A. M., Experimental discrimination of plowing friction and shear friction, Tribology Letters, Vol.25, No.2 (2007), pp.149-152.

Kesmez, Ö., Tamsü Selli, T., Tunalı, A., Akarsu, E., Akarsu, M. and Arpaç, E., Fingerprint resistant coatings for stainless steel substrates, Progress in Organic Coatings, Vol.112, (2017), pp.51-56.

Messaoud, W. B., Bueno, M.-A. and Lemaire-Semail, B., Relation between human perceived friction and finger friction characteristics, Tribology International, Vol.98, (2016), pp.261-269.

Nakanishi, Y., Nakashima, Y., Fujiwara, Y., Komohara, Y., Takeya, M., Miura, H. and Higaki, H., Influence of surface profile of Co-28Cr-6Mo alloy on wear behaviour of ultrahigh molecular weight polyethylene used in artificial joint, Tribology International, Vol.118, (2018), pp.538-546.

Psoma, S. D., Van der Wal, P. D., Frey, O., De Rooij, N. F. and Turner, A. P., A novel enzyme entrapment in SU-8 microfabricated films for glucose micro-biosensors, Biosensors and Bioelectronics, Vol.26, No.4 (2010), pp.15821587.

Saha, A. A., Mitra, S. K., Tweedie, M., Roy, S. and McLaughlin, J., Experimental and numerical investigation of capillary flow in SU8 and PDMS microchannels with integrated pillars, Microfluidics and Nanofluidics, Vol.7, (2009), pp.451-455.

Saito, Y., Okamoto, S., Miki, A., Inomata, H., Hidaka, T. and Kasai, H., Fabrication of microstructure on glass surface using micro-indentation and wet etching process, Applied Surface Science, Vol.254, (2008), pp.7243-7249.

Tokuhisa, H., Tsukamoto, S., Morita, S., Ise, S., Tomita, M. and Shirakawa, N., Fabrication of micro-textured surfaces for a high hydrophobicity by evaporative patterning using screen mesh templates, Applied Surface Science, Vol.400, (2017), pp.64-70.

Uhlmann, P., Frenzel, R., Voit, B., Mock, U., Szyszka, B., Schmidt, B., Ondratschek, D., Gochermann, J. and Roths, K., Research agenda surface technology: Future demands for research in the field of coatings materials, Progress in Organic Coatings, Vol.58, No.2-3 (2007), pp.122-126.

Wang, L., He, Y., Zhou, J. and Duszczyk, J., Effect of temperature on the frictional behaviour of an aluminium alloy sliding against steel during ball-on-disc tests, Tribology International, Vol.43, No.1-2 (2010), pp.299-306.

Yin, L., Baba, T. and Nakanishi, Y., Fracture-free surfaces of CAD/CAM lithium metasilicate glass-ceramic using microslurry jet erosion, Journal of the Mechanical Behavior of Biomedical Materials, Vol.80, (2018), pp.59-67.

Zhang, S., Rodriguez, U. A., Morales H. M., Zeng, X., and Van Der Heide, E., The role of the sliding direction against a grooved channel texture on tool steel: An experimental study on tactile friction, International Journal of Solids and Structures, Vol.56-57 (2015), pp.53-61

Zhou, W., Tang, J., Chen, H., Zhu, C. and Shao, W., A comprehensive investigation of plowing and grain-workpiece micro interactions on 3D ground surface topography, International Journal of Mechanical Sciences, Vol.144, (2018), pp.639-653. 